\title{
Modifying the temperature dependence of magnetic garnet film coercivity by etching
}

\author{
G. Vértesy and B. Keszei \\ Research Institute for Technical Physics and Materials Science, Hungarian Academy of Sciences, \\ H-1525 Budapest, P.O. Box 49, Hungary
}

(Received 10 May 1999; accepted for publication 2 September 1999)

\begin{abstract}
The temperature dependence of the domain-wall coercive field of epitaxial magnetic garnet films was modified in a defined temperature range by removing the surface layer of the films. Outside the given temperature range the coercivity versus temperature curve did not change. The result supports a model of coercivity according to which different sets of material imperfections are responsible for pinning the domain walls in different temperature regions. Appropriate processing of the samples enables some of the pinning sets to be modified independently of each other. (C) 1999 American Institute of Physics. [S0021-8979(99)08623-5]
\end{abstract}

\section{INTRODUCTION}

The temperature dependence of the domain-wall coercive field $H_{c w}$ was studied in magnetically uniaxial, liquidphase epitaxially grown, rare-earth garnet films, in the whole temperature range of the ferrimagnetic phase of the samples. ${ }^{1,2}$ A mathematical expression describing the temperature dependence of $H_{c w}$ was suggested, and conclusions were drawn about different types of pinning traps in the material. The measured domain-wall coercive field exhibited a steep decrease with increasing temperature $T$. The semilogarithmic plots of $H_{c w}(T)$ suggest that the drop is piece-wise exponential, with different slopes at different successive temperature regions. The temperature dependence of $H_{c w}$ was fitted to the exponential function

$$
H_{c w}=H_{c w o}^{i} \exp \left(-T / T_{i}\right),
$$

in each of its linear parts on a semilogarithmic plot, where $H_{c w o}^{i}$ and $T_{i}$ were the characteristic values for the $i$ th temperature range.

In explanation, it was suggested ${ }^{1,2}$ that different types of wall-pinning traps (material defects) coexist in the sample, each of them prevailing in one of the different temperature regions. The breaking points on the semilogarithmic plot of $H_{c w}(T)$ were identified by the limits of activity of one type of wall-pinning trap and the taking over of the next type. The existence and position of the temperature regions of $H_{c w}(T)$ with distinct breaking points in the semilogarithmic plot can be considered to be characteristic of each of the samples.

A survey of literature data and of data measured on a large number of our own samples was also carried out to show the general existence of this piece-wise exponential dependence and of the existence of the breaking points. ${ }^{3}$ This type of domain-wall coercive field temperature dependence was found in all the collected cases of the large family of epitaxial garnets (about 30 samples of more than 10 chemical compositions).

As was pointed out in Refs. 1-3, different sets of material imperfections are responsible for pinning of the domain walls; these material imperfections determine the $H_{c w}(T)$ curve in a certain range of temperature. However if these sets of material imperfections are independent of each other, it should be possible-at least in principle - to modify them independently of each other, with the result that modification of the $H_{c w}(T)$ curve takes place only in the corresponding temperature range. Because of this it can be expected that if the samples are appropriately processed, some of the pinning sets can be annihilated, generated, or at least modified which, in turn, could lead to modification of the temperature dependence of the domain-wall coercive field.

In the present work, to prove the above-mentioned assumption, and by this the whole model of the temperature dependence of coercivity, the surface layers of the magnetic films were removed, and the temperature dependence of $H_{c w}$ of the processed samples was measured. The results were compared with the temperature dependence of $H_{c w}$ of the original, nonprocessed samples.

\section{EXPERIMENTAL RESULTS}

Measurements were performed on three different magnetic garnet films, labeled A, B, and C. The films, each of different chemical composition were grown by liquid-phase epitaxy (LPE) on [111]-oriented gadolinium garnet (GGG) substrates. The samples exhibited large uniaxial anisotropy, which was perpendicular to the film plane, and superimposed over a small cubic one. The magnetization vector inside all domains was aligned perpendicular to the film plane. The basic parameters of the samples (see Table I) were determined at room temperature by standard methods of bubble garnet film characterization. ${ }^{4}$

Two methods for determining $H_{c w}$ were used, giving mutually equivalent results of $H_{c w}$. The low-frequency domain-wall oscillation method ${ }^{5}$ is based on the magnetooptical response of the domain system to an ac magnetic field perpendicular to the film plane. The ac field amplitude is slowly increased from zero and any domain-wall motion is detected photoelectrically. The field amplitude corresponding to the extrapolated start of the domain-wall motion is 
TABLE I. Chemical composition, room-temperature values of domain-wall coercive field $\left(H_{c w}\right)$, film thickness $(h)$, zero-field stripe domain period $\left(p_{0}\right)$, saturation magnetization $\left(4 \pi M_{s}\right)$, uniaxial anisotropy constant $\left(K_{u}\right)$, quality factor $(Q)$, and characteristic length $(l)$ of the measured garnet samples A-C.

\begin{tabular}{ccccccccc}
\hline \hline Sample & Composition & $H_{c w}(\mathrm{Oe})$ & $h(\mu \mathrm{m})$ & $p_{0}(\mu \mathrm{m})$ & $4 \pi M_{s}(\mathrm{G})$ & $K_{u}\left(\mathrm{erg} / \mathrm{cm}^{3}\right)$ & $Q$ & $l(\mu \mathrm{m})$ \\
\hline $\mathrm{A}$ & $(\mathrm{YSmCa})_{3}(\mathrm{FeGe})_{5} \mathrm{O}_{12}$ & 0.2 & 5.3 & 9.0 & 211 & 6400 & 4.2 & 0.47 \\
$\mathrm{~B}$ & $(\mathrm{YSm})_{3}(\mathrm{FeGa})_{5} \mathrm{O}_{12}$ & 0.4 & 4.9 & 9.7 & 191 & 15500 & 10.6 & 0.60 \\
$\mathrm{C}$ & $(\mathrm{YSmCaLu})_{3}(\mathrm{FeGe})_{5} \mathrm{O}_{12}$ & 0.3 & 2.8 & 6.4 & 338 & 36670 & 8.1 & 0.39 \\
\hline \hline
\end{tabular}

interpreted as $H_{c w}$. These experiments were performed in a purpose-built continuous-flow cryostat using liquid-nitrogen vapor to cool the sample to about $90 \mathrm{~K}$. The sample can also be heated to $500 \mathrm{~K}$ in the same holder. The other method measures specific minor hysteresis loops ${ }^{6}$ in a vibrating sample magnetometer. The magnetic moment of the sample is measured with the external magnetic field parallel to the easy-magnetization axis, i.e., perpendicular to the film plane. The onset of the irreversible domain-wall motion is detected in the measurement. Measurements were performed between 10 and $300 \mathrm{~K}$ on a vibrating sample magnetometer PAR model 155 with a helium cryostat. The results of these two measurement methods are identical with each other, as was shown in Ref. 6, because of this no distinction was made on the figures between points obtained by different methods.

The accuracy of the $H_{c w}$ values, especially in the hightemperature range, is crucial for the reliable interpretation of the results. In the low-temperature range $(20-150 \mathrm{~K})$ the accuracy is $\pm 0.2 \mathrm{Oe}$; in the middle of the temperature range $(150-300 \mathrm{~K})$ it is $\pm 0.1 \mathrm{Oe}$; in the high-temperature region (above $300 \mathrm{~K}$ ), the accuracy is estimated to be \pm 0.03 Oe. This relatively high accuracy was achieved by increasing the amplification of the ac method, and by repeated measurements. At every temperature value in the high-temperature region the coercivity was measured several times, and the average value was taken.

All three original (not etched) samples showed similar temperature dependence of the domain-wall coercive field. The $H_{c w}(T)$ curve is characterized by three pairs of $H_{c w o}^{i}$ and $T_{i}$ parameters, i.e., the $H_{c w}(T)$ curve consists of three linear segments in the semilogarithmic plot, with two distinct breaking points (see the solid line in Fig. 1).

Samples A and B were cut into several pieces. The surface layer of the epitaxial film was removed by chemical

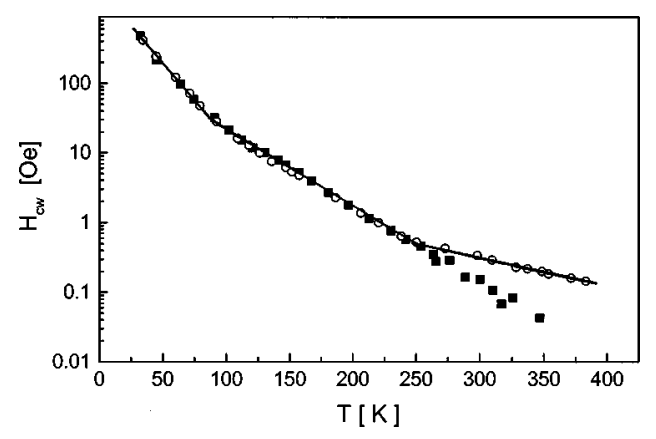

FIG. 1. Temperature dependence of domain-wall coercive field $H_{c w}$ measured on sample B. As-grown sample $(\bigcirc)$, and etched $(\Delta h=0.3 \mu \mathrm{m})$

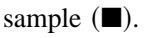

etching (except for a control piece of the sample) in hot $\left(150{ }^{\circ} \mathrm{C}\right)$ phosphoric acid to provide different remaining film thicknesses. The removed parts of the film thickness were $\Delta h=0.1,0.6,0.8$, and $1.4 \mu \mathrm{m}$ for sample A, and $\Delta h=0.3$, 0.7 , and $1.2 \mu \mathrm{m}$ for sample B. After chemical etching the domain-wall coercive field of every piece of both samples was measured again as a function of temperature.

In the case of sample $\mathrm{C}$, the $H_{c w}(T)$ curve was measured first on one piece of the original sample, then the same piece of the sample was chemically etched $(\Delta h=0.2 \mu \mathrm{m})$. After this procedure the $H_{c w}(T)$ curve was remeasured.

In all the etched cases of the three different samplesindependently of the value of $\Delta h$-the same result was found: the second, higher temperature breaking point disappeared, the whole $H_{c w}(T)$ curve could be characterized after the etching by only two pairs of $H_{c w o}^{i}$ and $T_{i}$ parameters instead of three.

The results of the measurements are illustrated in the full temperature range in Fig. 1, where the temperature dependence of $H_{c w}$ is seen on a semilogarithmic plot for the original, nonetched control piece of sample B (solid line, the measured values marked by " $\bigcirc$ ") and for the piece of the same sample from which a $0.3 \mu \mathrm{m}$ surface layer was removed (the measured values marked by ' $\square$ '). The same results were obtained on the other etched pieces of this sample, and also in the case of every piece of sample A, and in the case of sample $\mathrm{C}$ both before and after the etching. In Fig. 2 only the high-temperature range of the two pieces of sample $\mathrm{C}$ is shown, with more measured points, and the error bars are also indicated.

\section{DISCUSSION}

As clearly seen on Figs. 1 and 2, the removal of the surface layer has a considerable influence on the temperature

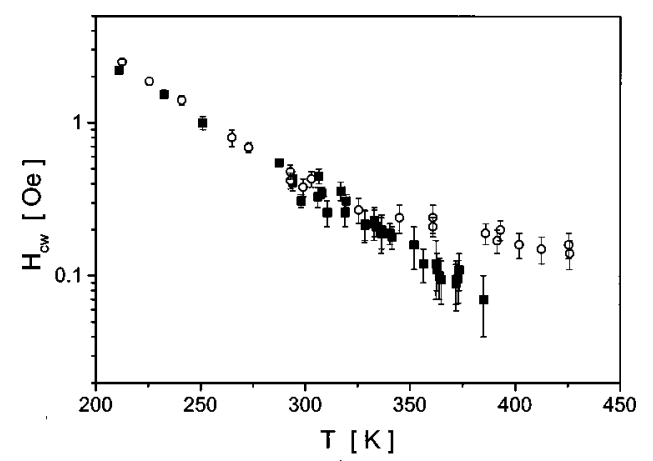

FIG. 2. Temperature dependence of domain-wall coercive field $H_{c w}$ measured on sample C. As-grown sample $(\bigcirc)$, and etched sample $(\boldsymbol{\square})$. 
dependence of domain-wall coercivity in the hightemperature region. As a result, one of the breaking points disappeared, the $H_{c w}(T)$ curve of the etched films can be described by two exponential segments instead of three. The effect does not depend on the thickness of the removed layer. The same phenomenon was observed after removing 0.1-1.4 $\mu \mathrm{m}$ of the film.

The coercivity depends on the thickness of the film, as can also be seen from Eq. (2). The thickness dependence of coercivity was investigated by a number of authors, ${ }^{7-9}$ and the $H_{c} \sim h^{-1}$ dependence was found to be valid. We also experienced a slight increase of the coercivity in the case of the larger $\Delta h$ values $(0.8-1.4 \mu \mathrm{m})$, but this change was not significant, compared with the original value of $H_{c w}$, and the effect resulted in a uniform shift of the $H_{c w}(T)$ curve, without any influence on the step-wise exponential character of the curve or on the breaking points in the semilogarithmic plot.

The result can be interpreted on the basis of the assumed existence of more mutually independent sets of material imperfections, which lead to domain-wall pinning. ${ }^{1}$ It was found that it is possible to modify the temperature dependence of the domain-wall coercivity of epitaxial magnetic garnet films by appropriate processing. This modification takes part only in a certain range of temperature; the other part of the $H_{c w}(T)$ curve remained unchanged. Our results serve as direct, empirical proof of the model. In such a way, the existence of different independent sets of material imperfections in the material was proved; these material imperfections are responsible for the pinning of the domain walls in a certain temperature range.

On the other hand, the effect of the film surface on the coercive properties was also shown. It was found that the surface of the film contributes to the domain-wall coercivity, probably because one of the sets of the material imperfections leads to domain-wall pinning. In the measured samples this is the main source of the room-temperature value of the measurable $H_{c w}$. It was also found-at least in the case of the surface removal - that the different sets of pinning traps, and together with them, the coercive properties of the material could be modified independently of each other by suitable processing of the sample. It also turned out that a very thin layer of the surface was responsible for the abovementioned domain-wall pinning, because after removing a very thin $(0.1 \mu \mathrm{m})$ surface layer, the coercive properties had been already modified, and after removing thicker layers, the same result was obtained.

Various possibilities for the change of coercivity due to chemical etching are taken into account and discussed. First, it was assumed that the rapid $150^{\circ} \mathrm{C}$ annealing applied during the chemical etching, was not responsible for the observed effect. This assumption was proved by an independent series of measurements, in which $150^{\circ} \mathrm{C}$ annealing was applied, without any measurable effect on the parameters of the garnet films.

Our experimental work indicated that we should take into account at least three possible sources of the observed high-temperature breaking point: (i) quality of the surface (roughness), (ii) difference between the chemical composi-

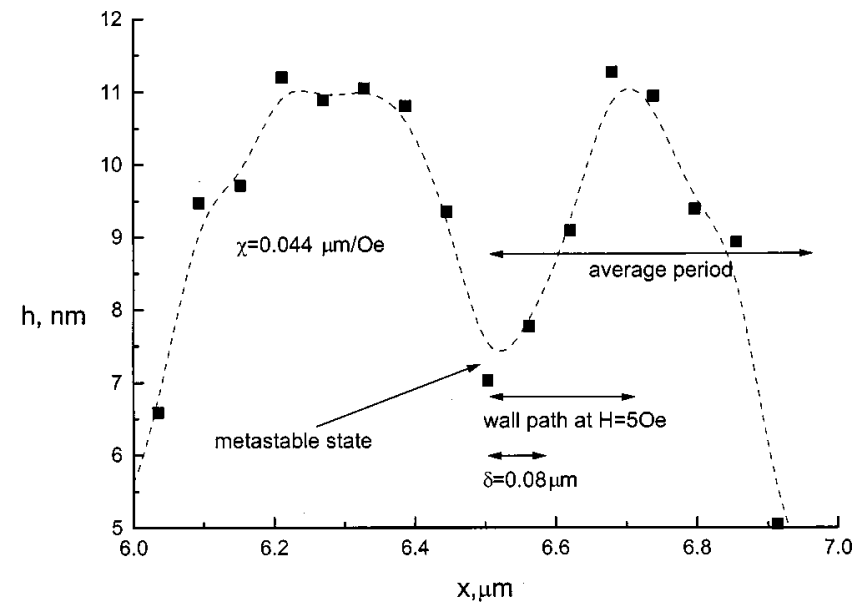

FIG. 3. Typical surface profile measured by AFM on sample B. Domainwall thickness, average period of film thickness fluctuations, susceptibility of the domain wall in $\mu \mathrm{m} / \mathrm{Oe}$, and wall travel path when field is increased by 5 Oe are shown.

tion of the thin surface layer and that of the inside of the whole sample, and (iii) stresses in the surface layer and/or in the whole sample.

To clarify the influence of the quality of the surface of our samples, we measured the surface roughness by Talystep as well as by atomic force microscopy (AFM) on the asprocessed and etched samples. No connection between the measured $H_{c w}(T)$ and the surface profile was found.

Independent measurements were taken to estimate the surface roughness. The surface profiles of the films were measured by atomic force microscopy, and then the results were used as an input for a micromagnetic model of the two-dimensional domain-wall motion process. The model was built assuming quasistatical motion of the flexible domain wall taking into account domain structure in the sample using mean-field approximation. ${ }^{10}$ These calculations showed that the surface roughness effects on the coercivity were prevented by the presence of the domain structure. If the domain structure was not taken into account during the calculations, the resulting coercivity was found to be less or comparable to the measured values. The reason for such behavior is the fact that the characteristic defect period on the surface profile is much greater than the distances traveled by domain walls during typical coercivity measurements (Fig. 3 ). Thus, domain-wall motion is almost reversible, with irreversibility stemming from a few places where the local distance between defects is small. It is emphasized that coercivity estimated using the one-dimensional approach may be large in this situation. For instance, taking the expression of Pardavi-Horváth ${ }^{11}$ based on Träuble ${ }^{12}$ and Kronmüller $^{13}$ statistical potential theory,

$$
H_{c w}=\frac{\pi}{M_{s}} \frac{\Delta h}{h d} \sigma\left(\frac{L}{2 d}\right)^{1 / 2}\left(\ln \frac{p_{0}}{2 \delta}\right)^{1 / 2},
$$

and substituting $4 \pi M_{s}=191 \mathrm{G}$, domain-wall width $\delta=0.08$ $\mu \mathrm{m}$, domain-wall energy density $\sigma=0.166 \mathrm{erg} / \mathrm{cm}^{2}$, equilibrium stripe period $p_{0}=9.2 \mu \mathrm{m}$, film thickness $h=4.4 \mu \mathrm{m}$, amplitude of the thickness fluctuations $\Delta h=10 \mathrm{~nm}$, and their 
period $d=2 \mu \mathrm{m}$, we can estimate the coercivity as being 0.09 Oe (when $L=0.05 \mu \mathrm{m}$ corresponds to our micromagnetic calculations) and it is larger for larger $L$. The parameter $L$ (length of the domain-wall segment moving as one single identity) is an artifact of the one-dimensional approach and can hardly be reasonably estimated.

The examined layers were prepared by the LPE method at a steady state. That is, the growth temperature, the substrate rotation rate, and the substrate position below the surface of the melt solution are constant, and for this reason the layer growth rate is also constant. ${ }^{14}$ This means that the chemical composition of the layer does not change during the layer growth process. However, at the end of the growth, the steady-state growth is discontinued by pulling out the substrate/layer from the melt, thus the layer growth rate also changes. This may produce a modification in the composition of the layer as the segregation coefficents of the cations (between the melt solution and solid) are not uniform. ${ }^{15}$ This indicates a stress between the "bulk" and this "secondary" surface layer which may, in turn, affect the domain-wall motion. Under the usual LPE growth conditions $(0.5-1 \mu \mathrm{m} / \mathrm{min}$ in steady-state growth rate, $15-20 \mathrm{~mm}$ in growth depth under the melt surface, and $30-40 \mathrm{~mm} / \mathrm{min}$ in pulling up rate), the thickness of this "secondary" surface layer is less than $0.5 \mu \mathrm{m}$.

To demonstrate these changes, we examined the chemical composition of the surface layers by the x-ray photoelectron spectroscopy (XPS) method (x-ray irradiation of the samples and measurement of the photoelectrons excited from the sample). Depending on the given element, the sensitivity of XPS is between 0.1 and 1 at. \%. The information depth of XPS is about 6-8 $\mathrm{nm}$ depending on the kinetic energy of photoelectrons in question. The spectra obtained showed no difference in percentage concentrations of basic elements $(\mathrm{Fe}, \mathrm{O}, \mathrm{Y}, \mathrm{Ca}, \ldots)$ in the virgin and etched samples. Thus, no measurable change in the chemical composition of the surface originated from the various sample treatments. The only noticeable difference in spectra shows itself in the background (although the differences in background are not correlated with sample processing) and can be attributed to the surface roughness of the film, which changes the scattering angle locally. Some pairs of samples show higher backgrounds for etched samples, and some for the virgin. This could be another indication that surface roughness itself does not give the main contribution to the coercivity of the given films at all temperatures.

The results of the XPS measurements seem to contradict the above assumption about the possible changes in the composition due to the secondary surface layer. However, the sensitivity of the measurement is insufficient to detect very small changes in the composition. It is known that the magnetic parameters, mainly uniaxial anisotropy, which has a large influence on the coercivity through the domain-wall parameters (domain-wall energy and thickness), are very sensitive to any small change in the composition, so it is possible that even very small differences in the chemical composition have a much larger influence on the magnetic parameters. A more likely explanation is - due to the modified growth conditions at the end of the growth process - that periodic fluctuations of $\mathrm{Sm}$ or $\mathrm{Ca}-\mathrm{Ge}$ or $\mathrm{Ga}$ content appear, without any measurable modification of the averaged chemical composition. Such fluctuations may cause a periodically modulated stress field, which leads to coercivity. ${ }^{16}$ Another possible effect might arise from the modification of the distribution of $\mathrm{Ge}$ and $\mathrm{Ga}$ ions between octahedral and tetrahedral sublattices: the nonmagnetic $\mathrm{Ge}$ and $\mathrm{Ga}$ ions have a preference for tetrahedral sites. In spite of the strong site selectivity of these ions, a certain proportion of them occupy octahedral sites. ${ }^{17}$ Chemical analysis is able to show the total amount of an element in the material, regardless of its distribution between the sublattices. However, from the viewpoint of magnetic properties, only substituting ions occupying tetrahedral sites are active, and any rearrangement of them between sublattices leads to a modification of magnetic properties.

As is well known, stresses exist in epitaxially grown magnetic garnet films because of the mismatch between substrate and magnetic layer. Another reason for the increase in some stresses could be the way of producing samples (process of removing them from the liquid, rate of cooling, etc.). It is reasonable to suppose that the mismatch stresses are greater at the interface of the substrate and magnetic layer, whereas stresses from the extraction procedure are more likely to be concentrated near to the surface of the sample. Stresses arising from the cooling of the sample after its production will probably be spread throughout the whole sample volume. The observed changes of $H_{c w}(T)$ after etching lead to the conclusion that etching could probably also release stresses in the surface layer, which might be in addition to the possible chemical changes (see above), thereby providing another reason for the decrease in $H_{c w}$. Values of possible effects from the releasing of stresses were estimated and found to be in good agreement with the observations. ${ }^{18}$

Finally, annealing of the just-grown layer takes place during the lifting out of the sample from the furnace. During the 25-35 min cooling period from about $930^{\circ} \mathrm{C}$ to room temperature, diffusion of oxygen into a thin surface layer may cause a change in coercivity.

\section{CONCLUSIONS}

Surface chemical etching of epitaxially grown magnetic garnet films modifies the $H_{c w}(T)$ curve, but only in a defined temperature range. The phenomenon was interpreted on the basis of a recent model of the temperature dependence of coercivity, where different types of pinning sets were assumed in the material.

The role of the surface was also related to the coercive properties. Several possible mechanisms that might influence the surface contribution to the coercivity were taken into account and analyzed, but based on the available measurement data, we could not decide which one was responsible for the surface coercivity. It is also possible that a combination of some of the processes mentioned might contribute to the coercive properties. 


\section{ACKNOWLEDGMENTS}

The authors are grateful to L. Belliard for AFM, to J. Zemek for XPS measurements, and to K. Metlov for the calculations. The work was supported by Hungarian Scientific Research Fund through Project No. T-026153.

${ }^{1}$ G. Vértesy, I. Tomáš, L. Pust, and J. Paces, J. Appl. Phys. 71, 3462 (1992).

${ }^{2}$ J. Kadlecová, K. Metlov, I. Tomáš, and G. Vértesy, J. Phys. IV 8, 307 (1998).

${ }^{3}$ G. Vértesy and I. Tomáš, J. Appl. Phys. 77, 6426 (1995).

${ }^{4}$ R. M. Josephs, AIP Conf. Proc. 10, 286 (1972).

${ }^{5}$ J. A. Seitchik, G. K. Goldberg, and W. D. Doyle, J. Appl. Phys. 42, 1272 (1971).

${ }^{6}$ G. Vértesy, L. Pust, and I. Tomáš, J. Phys. D 24, 1482 (1991).
${ }^{7}$ E. B. Moore, B. A. Calhoun, and K. Lee, J. Appl. Phys. 49, 1879 (1978).

${ }^{8}$ G. P. Vella-Coleiro, F. B. Hagedorn, S. L. Blank, and L. C. Luther, J. Appl. Phys. 50, 2176 (1979).

${ }^{9}$ J. C. Walling, J. Appl. Phys. 50, 7832 (1979).

${ }^{10} \mathrm{~K}$. Metlov (private communication).

${ }^{11}$ M. Pardavi-Horváth, IEEE Trans. Magn. MAG-21, 1694 (1985).

${ }^{12} \mathrm{H}$. Träuble, in Problem der Metallphysik, edited by A. Seeger (Springer, Berlin, 1966), Vol. II.

${ }^{13}$ H. Kronmüller, J. Magn. Magn. Mater. 24, 159 (1981).

${ }^{14}$ M. B. Small and R. Ghez, J. Cryst. Growth 43, 512 (1978).

${ }^{15}$ H. D. Jonker and W. Van Erk, J. Cryst. Growth 48, 131 (1980).

${ }^{16}$ M. Pardavi-Horváth, A. Cziráki, I. Fellegvári, G. Vértesy, J. Vandlik, and B. Keszei, IEEE Trans. Magn. MAG-20, 1123 (1984).

${ }^{17}$ S. L. Blank, J. W. Nielsen, and W. A. Biolsi, J. Electrochem. Soc. 123, 856 (1976).

${ }^{18}$ V. A. Zablotskii, J. Magn. Magn. Mater. 182, 31 (1998). 